\title{
Study of Coating Geometries and Photoluminescence Properties of Metal Nanoparticles/Graphite Composites
}

\author{
Pasquale Barone, Marianna Barberio, Fabio Stranges, and Fang Xu \\ Biology, Ecology and Earth Science Department and Physics Department, University of Calabria, 87036 Rende, Italy \\ Correspondence should be addressed to Marianna Barberio; marianna.barberio@fis.unical.it
}

Received 30 December 2013; Accepted 30 March 2014; Published 17 April 2014

Academic Editor: Demeter Tzeli

Copyright (C) 2014 Pasquale Barone et al. This is an open access article distributed under the Creative Commons Attribution License, which permits unrestricted use, distribution, and reproduction in any medium, provided the original work is properly cited.

In this work we present the results of a study of growth and characterization of metal nanoparticles (Ag, Au, and Co)/carbon surfaces. The nanoparticles grew by laser ablation technique and their dimensions were controlled by light scattering study and AFM microscopy before their insertion on graphite surface. Nanoparticles appear randomly disposed on carbon surfaces aggregating to form big particles only in the case of silver. The different behavior of metal nanoparticles on carbon surface was explained in terms of different metal wetting of surface, in agreement with previous theoretical results of He et al. Chemical information, obtained by X-ray photoelectron spectroscopy, indicated that the doping process is a simple physisorption while the interfacial interaction between particles and carbon layers causes local defects in graphite structure and the appearance of a strong photoluminescence signal for all composites. Moreover, the visible optical absorption decreases about $10 \%$ indicating the progressive metallization of carbon surface.

\section{Introduction}

Carbon nanotubes (CNT) and graphene have attracted considerable interest in scientific community for their use in the fabrication of nanoelectronics devices as field effect transistors or nanodiodes. In these frameworks the interaction between metals and carbon based substrates at nanometric scale is of critical importance in determining device characteristics [1]. In fact, unlike silicon devices, the electrodes on carbon structures are made up of metals and so the control of contact resistance (including coating geometries and interfacial interactions) is strategic [1].

The study of interfacial properties and coatings geometry between different carbon surfaces and metals is so fundamental [1]. Various experiments have been carried out to investigate the contact between different metals and carbon structures [1-3]. As example the metal coating geometries on CNT are closely related to the contact resistance [1]. Coating of metals such as $\mathrm{Ti}, \mathrm{Pd}$, and Ni lead to the formation of continuous or quasicontinuous layers on the CNT indicating firm adsorption and low contact resistance, while metals such as $\mathrm{Fe}, \mathrm{Al}$, and $\mathrm{Au}$ lead to the construction of isolated clusters indicating weak interaction and large contact resistance $[1$, 4-7]. Studies based on density functional theory attribute the metallic coating properties to the metal-carbon binding energy $[6,7]$, while study on traditional nucleation and wetting theories indicate in the different metal wetting of carbon surfaces the different coating geometries [1].

Moreover, the combination between a carbon nanostructure and metal nanoparticles is much interesting because metal nanoparticles exhibit very important properties that can be improved by the mix with carbon based nanomaterials [1, 8-17]. In our previous work [18], we demonstrate that metal nanoparticles (growth by laser ablation techniques and afterwards inserted in carbon nanotubes networks) are distributed on carbon surface to forming coatings geometries that are uniquely related to wetting between carbon and metals. We do not observe formation of chemical bonds between tubes and nanoparticles but the introduction of several local defects on CNT structures which causes strong changes in morphology and the appearance of a strong photoluminescence signal in visible region [18].

Here we extend the study of interaction between metal nanoparticles (both noble and transitions metals- $\mathrm{Au}, \mathrm{Ag}$, 
and Co) and carbon surfaces (amorphous graphite and highly oriented pyrolytic graphite (HOPG)). metal nanoparticles were grown dimensionally controlled and inserted on carbon structures with the same process used for metal/carbon nanotubes nanocomposites in [18]. The obtained samples were characterized morphologically, chemically, and optically using AFM microscopy, X-ray photoelectron spectroscopy, photoluminescence spectroscopy, and optical absorptions measurements in visible regions. The obtained results are identical for both amorphous and oriented graphite (in the following will be showed only the results for HOPG).

\section{Materials and Methods}

2.1. Materials. Amorphous graphite and high oriented pyrolytic graphite (HOPG) were purchased from Goodfellow and they have a nominal purity greater than 99\%. AFM images in Figure 1 for pristine sheets indicate that the samples are smooth and without presence of external impurities. Moreover, the amorphous graphite appears as a random superposition of separate foils while in HOPG carbon foils are uniformly disposed.

Metal nanoparticles were produced by laser ablation (LA) of a metal plate in acetone (aqueous solution 99.5\%). The metal plate (>99.99\%) was vertically placed in a glass vessel filled with $25 \mathrm{~mL}$ of acetone and irradiated for several minutes by the first harmonic (1064 nm) of a Quanta-Giant series 710 Nd:YAG laser operating at $10 \mathrm{~Hz}$. The spot size of the laser beam on the surface of the metal plate is about $7-8 \mathrm{~mm}$ and the power of laser is fixed at $600 \mathrm{~mJ} /$ pulse. During irradiation of the laser beam, the solution, initially transparent, gradually turns colored, indicating the formation of nanoparticles in solution [18, 19]. After LA, the solution is sonicated for 30 minutes to prevent the self-aggregation of the nanoparticles suspended in acetone and the formation of macrocluster. The dimensions of the deposited particles after sonication are identical to those produced in solution during LA (about $40 \mathrm{~nm}$ ). The particle dimensions during LA were controlled monitoring the optical absorbance following the Kelly plasmonic theory [19] and subsequently verified by AFM microscopy (a drop of nanoparticles solution was deposed on a pure copper sheet (pure NP sample) and observed by microscopes). Metal nanoparticles HOPG (graphite) composites were obtained positioning the carbon sheets in the vessel glass containing the solution after sonication and then the solution was heated up to complete solvent evaporation on a hot plate at $180^{\circ} \mathrm{C}$.

2.2. Methods. Morphologic information of our composites has been obtained by a Bruker AFM microscope (ICON Bruker) working in tapping mode. The images have a resolution of $512 \times 512$ lines and are acquired at scanning rate of about $1 \mathrm{~Hz}$. The obtained images were processed with the Nanoscope software (provided by Bruker) to evaluate size distribution of particle dimensions.

Chemical and optical information on composites was obtained by photoluminescence spectroscopy (PL), X-ray photoelectron spectroscopy (XPS), and optical absorption measurements in all the visible range (400-800 nm).

Photoluminescence (PL) and optical absorption measurements were taken by an Olympus microscope (HoribaJobyn Yvon) mounting objectives of 10x, 50x, and 100x magnifications. The microscope is equipped by a $375 \mathrm{~nm}$-laser source with a power of $15 \mathrm{~mW}$, for PL, a white lamp (Energetiq LDLS, laser driven light source), for absorption, and by a Triax 320 (Horiba-Jobyn Yvon) spectrometer working in the 200-1500 $\mathrm{nm}$ range.

We measured directly the reflected spectrum $\left(I_{r}(\lambda)\right)$ and obtained, by simply assuming the transmittance to be null for bulk samples, the reflectance $(r(\lambda))$ and the absorbance $(\varepsilon(\lambda))$ as a function of wavelength by the relation

$$
\begin{gathered}
r(\lambda)=\frac{I_{r}(\lambda)}{I_{s}(\lambda)}, \\
\varepsilon(\lambda)=1-r(\lambda),
\end{gathered}
$$

where $I_{s}(\lambda)$ is the source spectrum.

XPS measurements, taken on all pure and composite samples, were conducted in a UHV chamber equipped for standard surface analysis with a pressure in the range of $10^{-9}$ torr. Nonmonochromatic Mg-K $\alpha$ X-ray $(h v=1253.64 \mathrm{eV})$ was used as excitation source. The XPS spectra were calibrated with the C1s peak of a pure carbon sample (energy position $284.6 \mathrm{eV}$ ). All XPS spectra have been corrected for analyzer transmission and the background has been subtracted using the straight line subtraction mode. Moreover, the XPS data were fitted assuming a Gaussian distribution of XPS-lines.

\section{Results and Discussion}

The AFM images of composites in Figure 1 and the particle dimensions histograms in Figure 2 (showed only for HOPG sample) show a very different morphology of nanocomposites obtained by noble or transitions NPs in either amorphous or oriented graphite.

The sample with silver nanoparticles shows the formation of big aggregates randomly distributed on sheet surface and with average dimensions of about $77 \mathrm{~nm}$. Gold nanoparticles, with dimensions of about $25 \mathrm{~nm}$, cover randomly all the sheet surface while cobalt nanoparticles are arranged either to form big aggregates or isolated particle with little particles of about $22 \mathrm{~nm}$. As visible in the left panel of Figure 1, Co nanoparticles with dimensions of about $22 \mathrm{~nm}$ are randomly disposed on HOPG surface, while only one isolated aggregate (with dimensions of about $200 \mathrm{~nm}$ ) is visible. These results are in agreement with those obtained for interaction between metal nanoparticle and carbon nanotubes in our previous work [18] and based on theoretical calculations of He et al. [1], confirming the different behavior of noble and transitions metals on carbon surfaces. So, we can definitively conclude that the different behavior of metals is determinate by their different wetting properties on carbon structures, in agreement with the theoretical work in [1]. He et al., in fact, have recently theoretically demonstrated [1] that the metal surface energies (defined as the difference between the total 

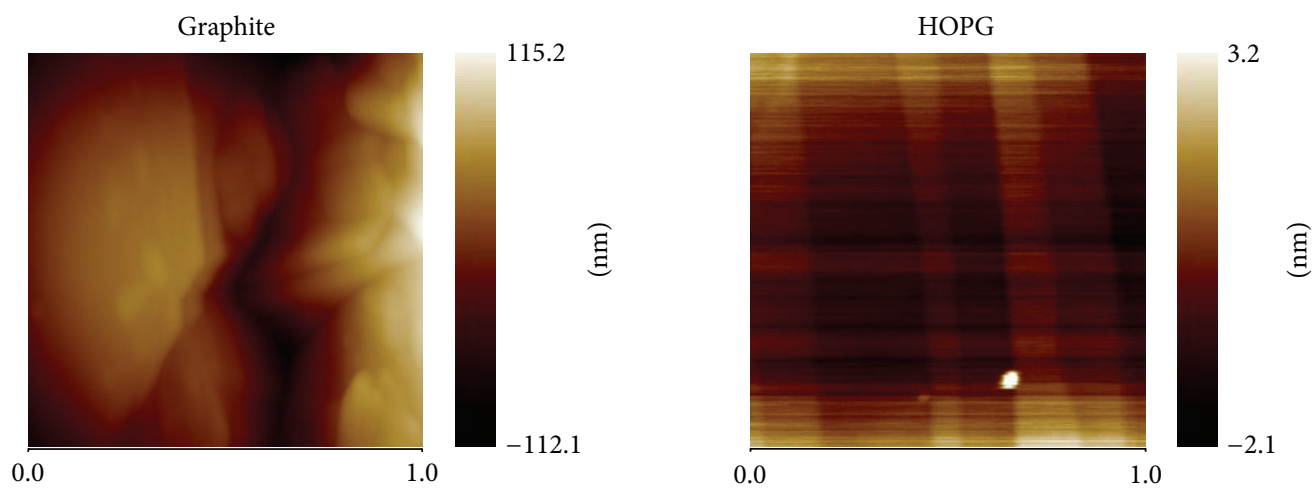

Height sensor $(\mu \mathrm{m})$

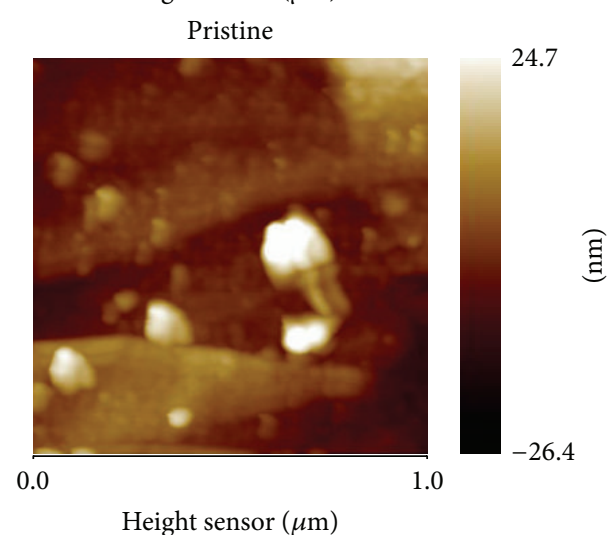

Height sensor $(\mu \mathrm{m})$

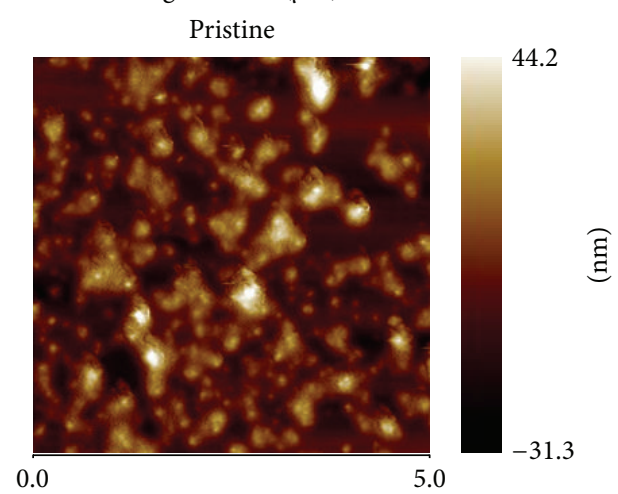

ight sensor $(\mu \mathrm{m})$

Ag

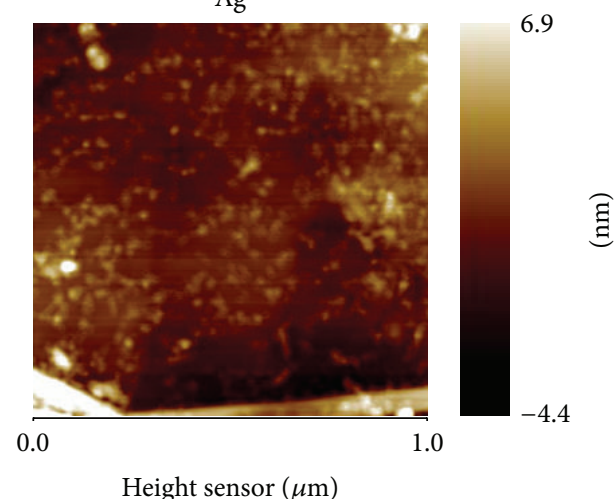

Height sensor $(\mu \mathrm{m})$

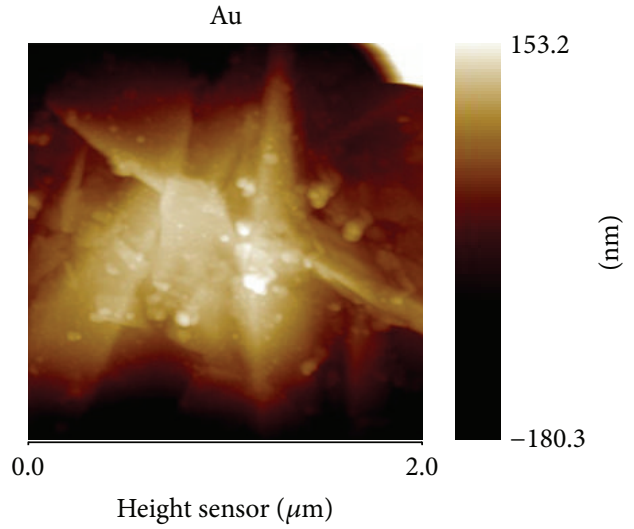

Co

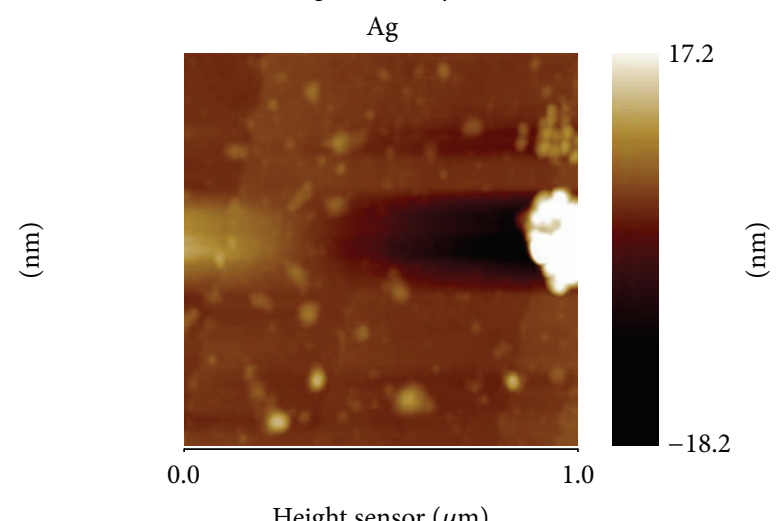

Height sensor $(\mu \mathrm{m})$

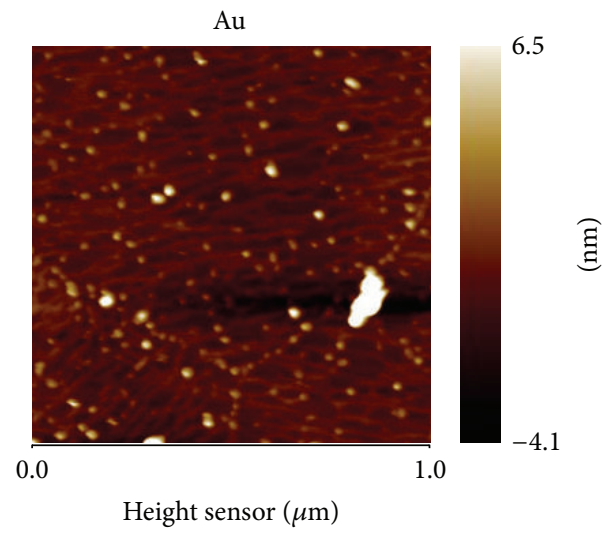

Co

FIGURE 1: AFM images for pristine amorphous and orientated graphite. The label under each image indicates the metal nanoparticle. 

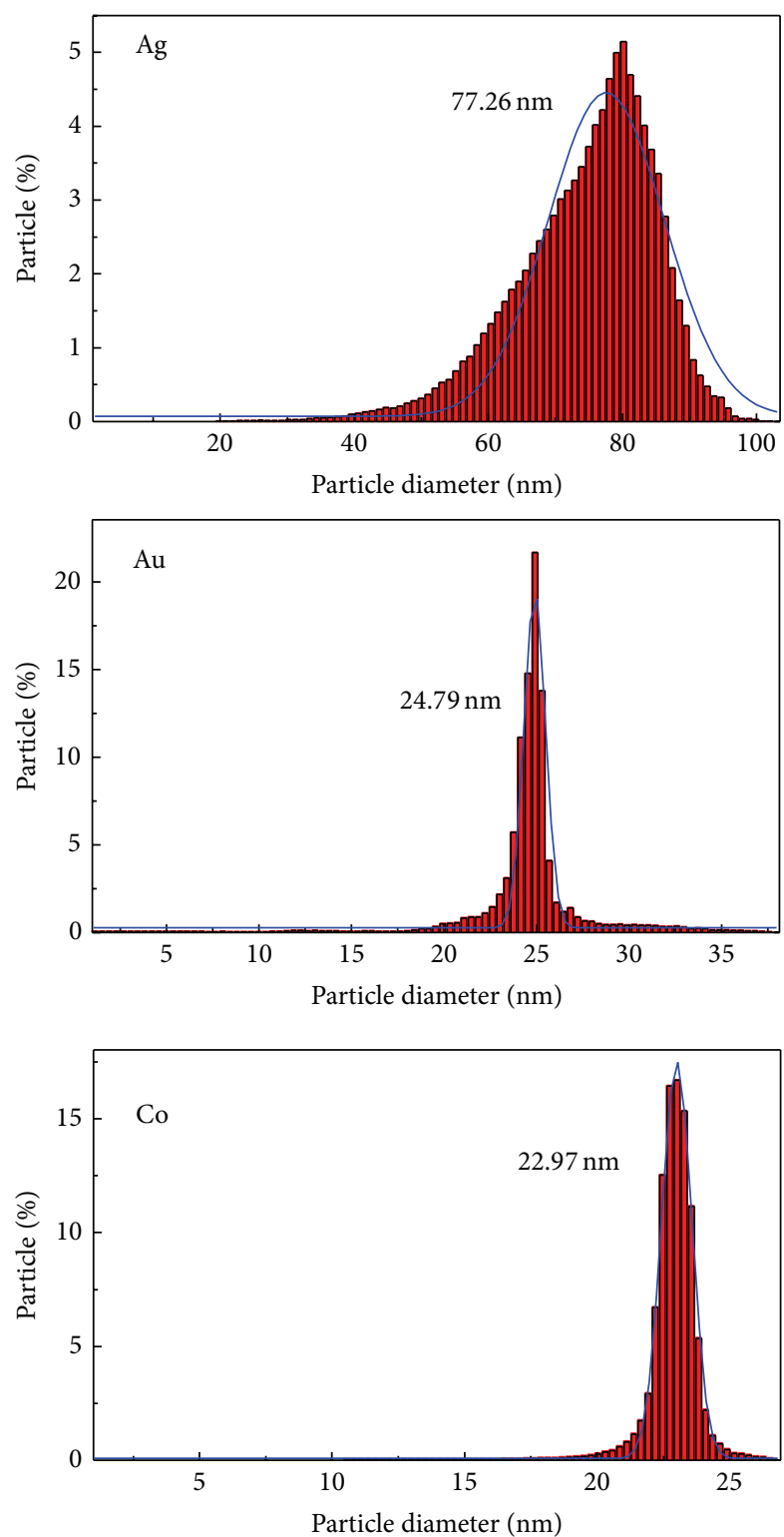

FIGURE 2: Histograms of particle dimensions distribution for each nanoparticle on HOPG surfaces obtained processing the AFM images by Nanoscope software (Bruker).

energy of a metal slab and the total number of atoms with energy per atom) and the metal-carbon interfacial energies are of critical importance to determine the nanoparticle arrangement on carbon surfaces. In their work He et al. calculated the above energies for metals atoms on single wall carbon nanotube surfaces. We can, so, extend their theoretical results on interactions between metal's atoms and SWNT also at atom aggregates (nanoparticles) on different carbon surfaces (multiwalled carbon nanotubes [18], amorphous graphite, and HOPG). He et al. suggest that for low cohesion energy, the metal atoms have the tendency to pile up and form isolated clusters. While for low interfacial energy the metal can be adsorbed on the carbon surface and tend to spread over. The wetting property of metals is so-determined by their

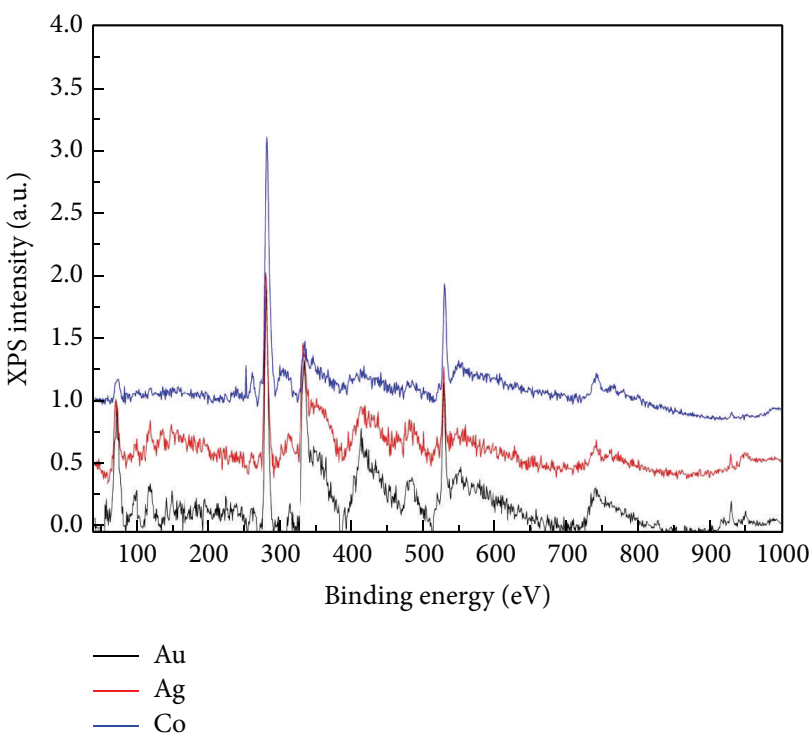

FigURE 3: XPS spectra for nanoparticle/HOPG composites.

TABLE 1: Element's amount on sample surface and XPS main line position evaluated from XPS data.

\begin{tabular}{|c|c|c|c|}
\hline \multicolumn{3}{|c|}{$\begin{array}{c}\text { Element's amount } \\
(\%)\end{array}$} & $\begin{array}{l}\text { XPS main line } \\
\text { position }(\mathrm{eV})\end{array}$ \\
\hline \multicolumn{4}{|l|}{ Ag/HOPG } \\
\hline Carbon & 91.87 & $\mathrm{C}$ 1s & $284.5 / 287.2$ \\
\hline Oxygen & 7.26 & O 1s & 531.5 \\
\hline Silver & 0.87 & $\operatorname{Ag} 3 d 3 / 2$ & 374.3 \\
\hline \multicolumn{4}{|l|}{$\mathrm{Au} / \mathrm{HOPG}$} \\
\hline Carbon & 87.52 & $\mathrm{C} 1 \mathrm{~s}$ & $284.2 / 286.7$ \\
\hline Oxygen & 11.80 & O 1s & 530.9 \\
\hline Golden & 0.67 & $\mathrm{Au} 4 \mathrm{f} 7 / 2$ & 83.62 \\
\hline \multicolumn{4}{|l|}{$\overline{\mathrm{Co} / \mathrm{HOPG}}$} \\
\hline Carbon & 91.4 & $\mathrm{C}$ 1s & $284.3 / 286.9$ \\
\hline Oxygen & 6.22 & $\mathrm{O} 1 \mathrm{~s}$ & 531.2 \\
\hline Cobalt & 2.34 & Co $2 p$ & 778.1 \\
\hline
\end{tabular}

combination action. Theoretical data in [1] indicated that noble metal ( $\mathrm{Au}$ and $\mathrm{Ag}$ ) have the high cohesion energy and low interfacial energy with tube suggesting that these metals rapidly diffuse on surface forming isolated cluster or covering the entire surface. For all transition metals, instead, the interfacial energy is high indicating that the interaction between tube and metal is stronger than metal-metal bulk interaction so the NPs can uniformly disperse on tube surface.

Chemical data on composites were obtained by XPS measurements. For all samples XPS spectra indicate the only presence of carbon, oxygen, and NP on sample surface (Figure 3), confirming the cleanness of surface and the absence of chemical impurities incorporated in growth process.

The XPS main position of all elements on sample surfaces (summarized in Table 1 for only HOPG) indicates the absence of chemical bonds between carbon nanotubes and metals specifying the van deer Waals nature of bonding between particle and carbon and the physisorption character of 


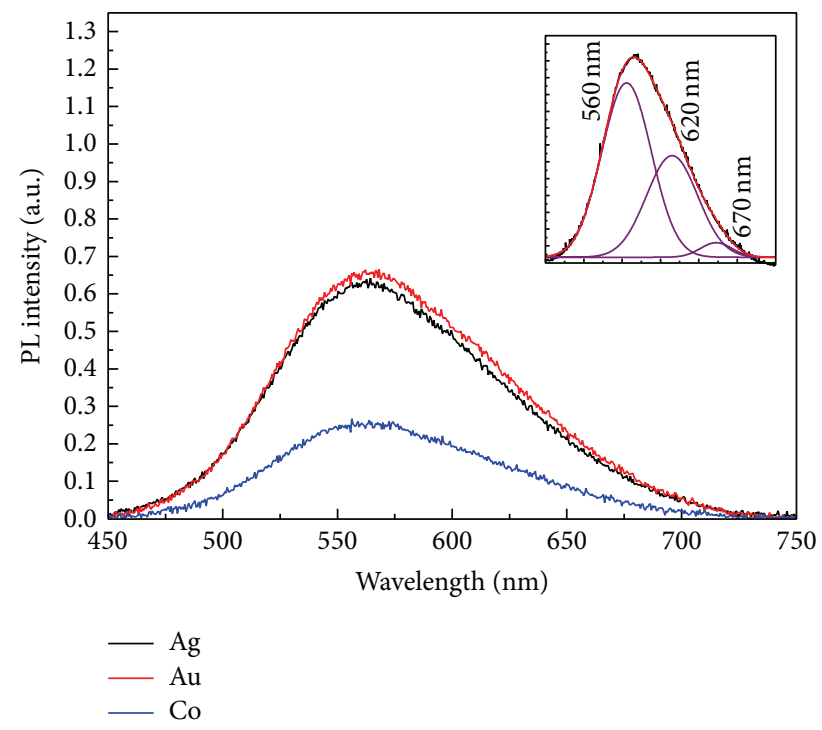

Figure 4: PL spectra for nanoparticle/HOPG composites. Inset: Gaussian spectrum deconvolution.

particle absorption on sheet surface. The $\mathrm{Cls}$ line exhibits two lines at about $284 \mathrm{eV}$ ( $\mathrm{C}$ main line indicating $\mathrm{C}-\mathrm{C}$ bonding) and $287 \mathrm{eV}$ (indicating the presence of $\mathrm{C}-\mathrm{O}$ bonds) [20]. The Ols line is, everywhere, symmetric and centered at about $531 \mathrm{eV}$ confirming the presence of $\mathrm{C}-\mathrm{O}$ bonding on surfaces [20]. All the metals exhibit the main atomic lines [20] without chemical shifts related to formation of chemical bonds between metal and $\mathrm{C}$ but indicating the presence of metal oxide formation. In particular, the position of main metallic XPS line indicates the formation of $\mathrm{Ag}-\mathrm{O}$ and $\mathrm{Co}-$ $\mathrm{O}$ chemical bonds (suggesting the presence of both $\mathrm{AgO}$ and $\mathrm{Ag}_{2} \mathrm{O}_{3}$ oxides for silver and $\mathrm{CoO}$ for cobalt).

Photoluminescence measurements in Figure 4 indicate the origin of a strong visible luminescence for all composites with a great effectiveness for samples obtained with gold and silver. All the PL spectra present two main bands centered at about 560 and $620 \mathrm{~nm}(2.2$ and $1.99 \mathrm{eV})$ and a loss intense band at $670 \mathrm{~nm}(1.84 \mathrm{eV})$. These structures indicate that the interaction between carbon surface and metal nanoparticles causes changes in graphite electronic band opening locally a band gap (caused by the defect introduced by nanoparticles in graphite structure) and favoring the visible transitions absent in pure graphite, as already observed in our previous work for carbon nanotube [18].

Optical absorption measurements in Figure 5 indicated a decrease of absorption of about $10 \%$ in all visible regions for all samples, indicating the progressive metallization of sample caused by the uniform coverage of nanoparticles on surfaces.

\section{Conclusions}

In this work we present the results of a study of interaction between nanoparticle and carbon surfaces which extend and conclude our previous study of interaction of metal nanoparticle and carbon nanotubes [18]. The metal nanoparticles

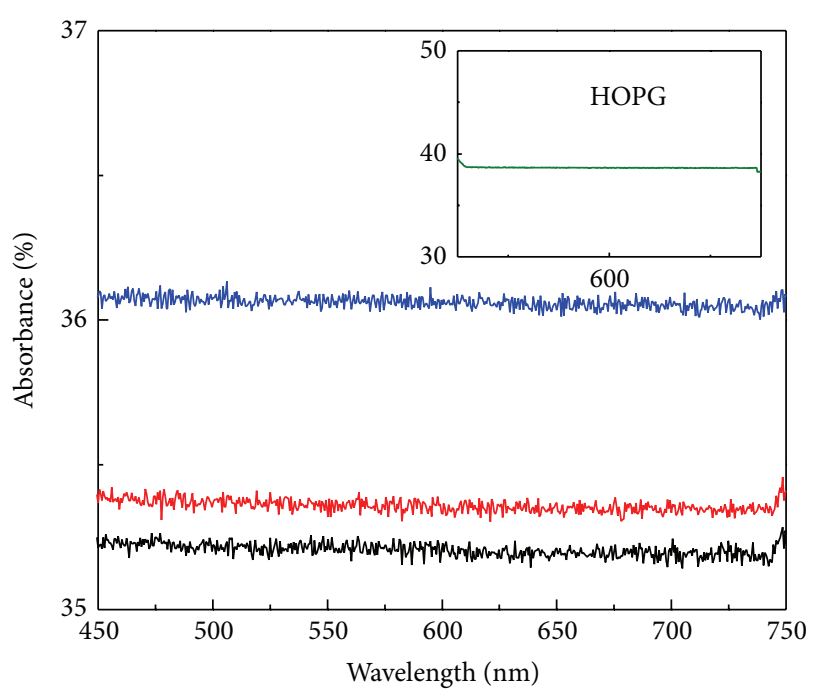

(a)

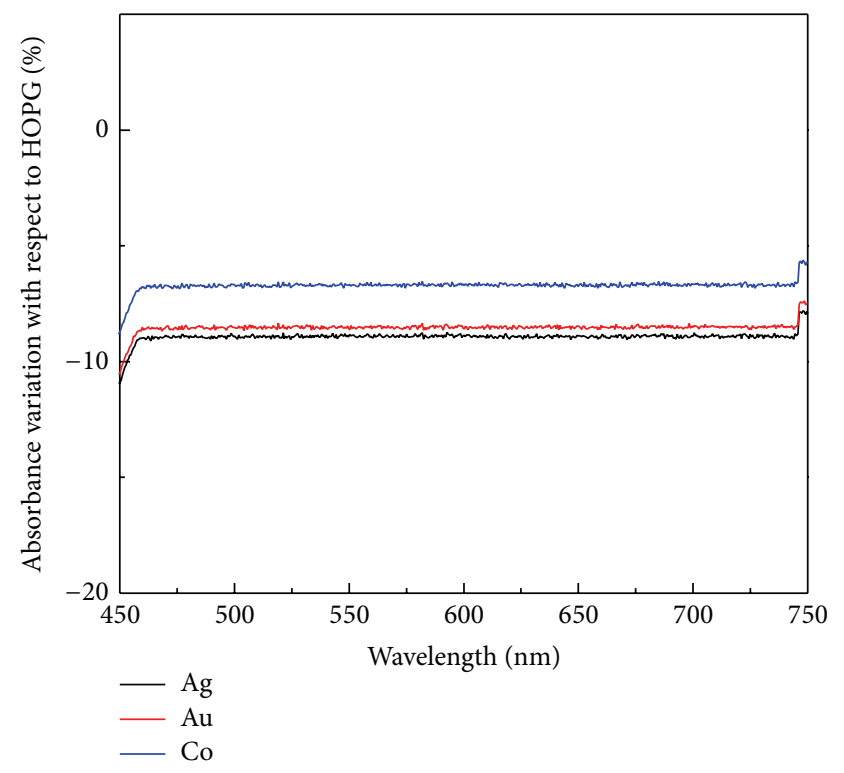

(b)

FIGURE 5: Optical absorption in visible region for nanoparticle/HOPG composites (a) and HOPG (inset in (a)). Variation of optical absorption with respect to pure HOPG (b).

(gold, silver, and cobalt) were obtained by laser ablation in acetone solution and their dimensions were controlled before the inclusion on carbon surfaces with Kelly scattering theory and AFM characterization as described in [18]. The experiment was conducted on both oriented and amorphous graphite layers. The results confirm what has been achieved for carbon nanotube. The nanoparticles are randomly disposed on carbon surfaces and aggregates to form big particles only in the case of silver. The different behavior of metals on carbon surface was explained (as in the case of carbon nanotube) in terms of different metal wetting of carbon surface.

The doping process is a simple physisorption; the XPS data in fact do not show formation of chemical bonds 
between carbon and metals. Furthermore, the interfacial interaction between particles and carbon layers (due to only van deer Waals interactions) causes local defects in graphite structure indicated by the presence of a strong photoluminescence signal for all composites. Moreover, we note a decrease of about $10 \%$ in visible optical absorption indicating the progressive metallization of carbon surface.

\section{Conflict of Interests}

The authors declare that there is no conflict of interests regarding the publication of this paper.

\section{Acknowledgment}

This work has been funded from the European projects PON 042a_E "Res Novae" and PON01_02140 “COMAS".

\section{References}

[1] Y. He, J. Zhang, Y. Wang, and Z. Yu, "Coating geometries of metals on single-walled carbon nanotubes," Applied Physics Letters, vol. 96, Article ID 063108, 2010.

[2] Y. Zhang, N. W. Franklin, R. J. Chen, and H. Dai, "Metal coating on suspended carbon nanotubes and its implication to metaltube interaction," Chemical Physics Letters, vol. 331, pp. 35-41, 2000.

[3] Z. Chen, J. Appenzeller, J. Knoch, Y.-M. Lin, and P. Avouris, "The role of metal-nanotube contact in the performance of carbon nanotube field-effect transistors," Nano Letters, vol. 5, no. 7, pp. 1497-1502, 2005.

[4] S. Inoue and Y. Matsumura, "Molecular dynamics simulation of metal coating on single-walled carbon nanotube," Chemical Physics Letters, vol. 464, pp. 160-165, 2008.

[5] N. Park, D. Sung, S. Lim, S. Moon, and S. Hong, "Realistic adsorption geometries and binding affinities of metal nanoparticles onto the surface of carbon nanotubes," Applied Physics Letters, vol. 94, Article ID 073105, 2009.

[6] A. Maiti and A. Ricca, "Metal-nanotube interactions-binding energies and wetting properties," Chemical Physics Letters, vol. 395, pp. 7-11, 2004.

[7] E. Fortunati, F. D’Angelo, S. Martino, A. Orlacchio, J. M. Kenny, and I. Armentano, "Carbon nanotubes and silver nanoparticles for multifunctional conductive biopolymer composites," Carbon, vol. 49, no. 7, pp. 2370-2379, 2011.

[8] G. D. Liang, S. P. Bao, and S. C. Tjong, "Microstructure and properties of polypropylene composites filled with silver and carbon nanotube nanoparticles prepared by meltcompounding," Materials Science and Engineering B, vol. 142, no. 2-3, pp. 55-61, 2007.

[9] P. C. Ma, B. Z. Tang, and J.-K. Kim, "Effect of CNT decoration with silver nanoparticles on electrical conductivity of CNTpolymer composites," Carbon, vol. 46, no. 11, pp. 1497-1505, 2008.

[10] J. Sandler, M. S. P. Shaffer, T. Prasse, W. Bauhofer, K. Schulte, and A. H. Windle, "Development of a dispersion process for carbon nanotubes in an epoxy matrix and the resulting electrical properties," Polymer, vol. 40, no. 21, pp. 5967-5971, 1999.

[11] L. B. Da Silva, S. B. Fagan, and R. Mota, "Ab initio study of deformed carbon nanotube sensors for carbon monoxide molecules," Nano Letters, vol. 4, no. 1, pp. 65-67, 2004.
[12] D. M. Guldi, G. M. A. Rahman, M. Prato, N. Jux, S. Qin, and W. Ford, "Single-wall carbon nanotubes as integrative building blocks for solar-energy conversion," Angewandte Chemie International Edition, vol. 44, no. 13, pp. 2015-2018, 2005.

[13] H. C. Choi, M. Shim, S. Bangsaruntip, and H. Dai, "Spontaneous reduction of metal ions on the sidewalls of carbon nanotubes," Journal of the American Chemical Society, vol. 124, no. 31, pp. 9058-9059, 2002.

[14] J. Chen, H. Liu, W. A. Weimer, M. D. Halls, D. H. Waldeck, and G. C. Walker, "Noncovalent engineering of carbon nanotube surfaces by rigid, functional conjugated polymers," Journal of the American Chemical Society, vol. 124, no. 31, pp. 9034-9035, 2002.

[15] J. Du, L. Fu, Z. Liu et al., "Facile route to synthesize multiwalled carbon nanotube/zinc sulfide heterostructures: optical and electrical properties," Journal of Physical Chemistry B, vol. 109, no. 26, pp. 12772-12776, 2005.

[16] R. Sepahvand, M. Adeli, B. Astinchap, and R. Kabiri, "New nanocomposites containing metal nanoparticles, carbon nanotube and polymer," Journal of Nanoparticle Research, vol. 10, no. 8, pp. 1309-1318, 2008.

[17] F. Xin and L. Li, "Decoration of carbon nanotubes with silver nanoparticles for advanced CNT/polymer nanocomposites," Composites A, vol. 42, no. 8, pp. 961-967, 2011.

[18] M. Barberio, P. Barone, F. Stranges, A. Romano, F. Xu, and A. Bonanno, "Carbon nanotubes/metal nanoparticle based nanocomposites: improvements in visible photoluminescence emission and hydrophobicity," Optics and Photonics Journal, vol. 3, pp. 34-40, 2013.

[19] K. L. Kelly, E. Coronado, L. L. Zhao, and G. C. Schatz, "The optical properties of metal nanoparticles: the influence of size, shape, and dielectric environment," Journal of Physical Chemistry B, vol. 107, no. 3, pp. 668-677, 2003.

[20] NIST XPS data base, http://srdata.nist.gov/xps/selEnergyType .aspx. 

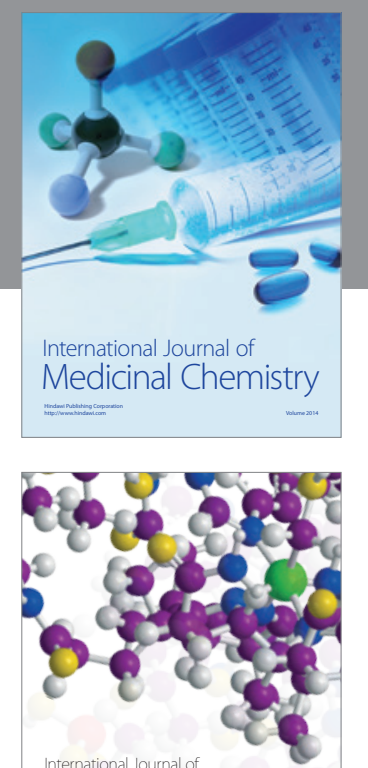

\section{Carbohydrate} Chemistry

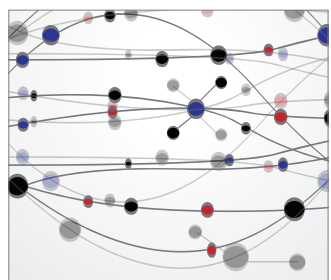

The Scientific World Journal
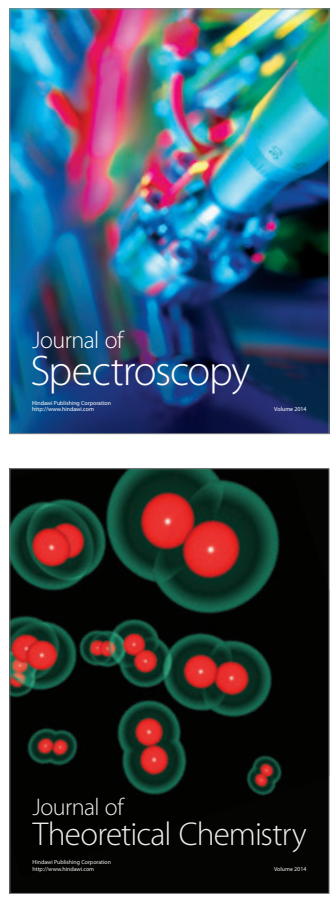
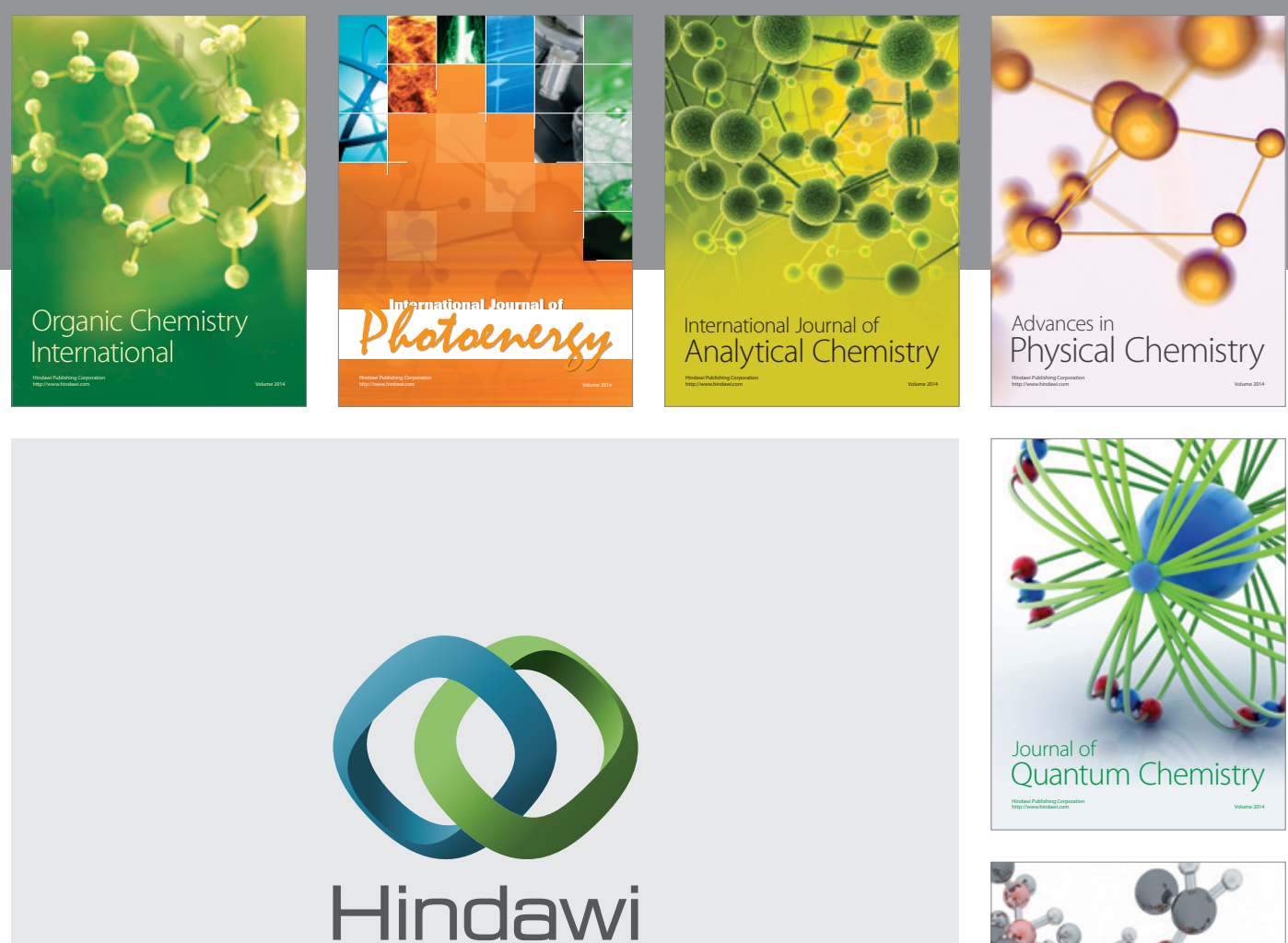

Submit your manuscripts at

http://www.hindawi.com

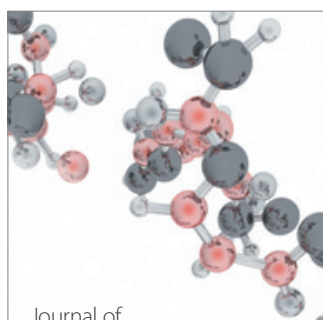

Analytical Methods

in Chemistry

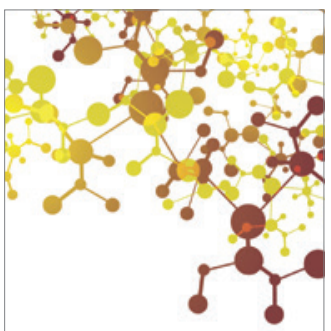

Journal of

Applied Chemistry

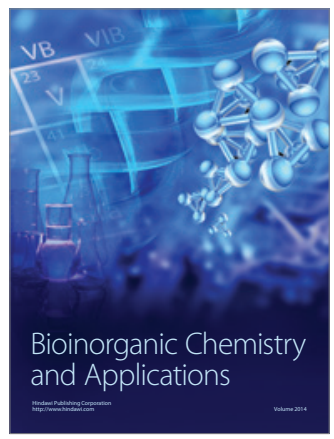

Inorganic Chemistry
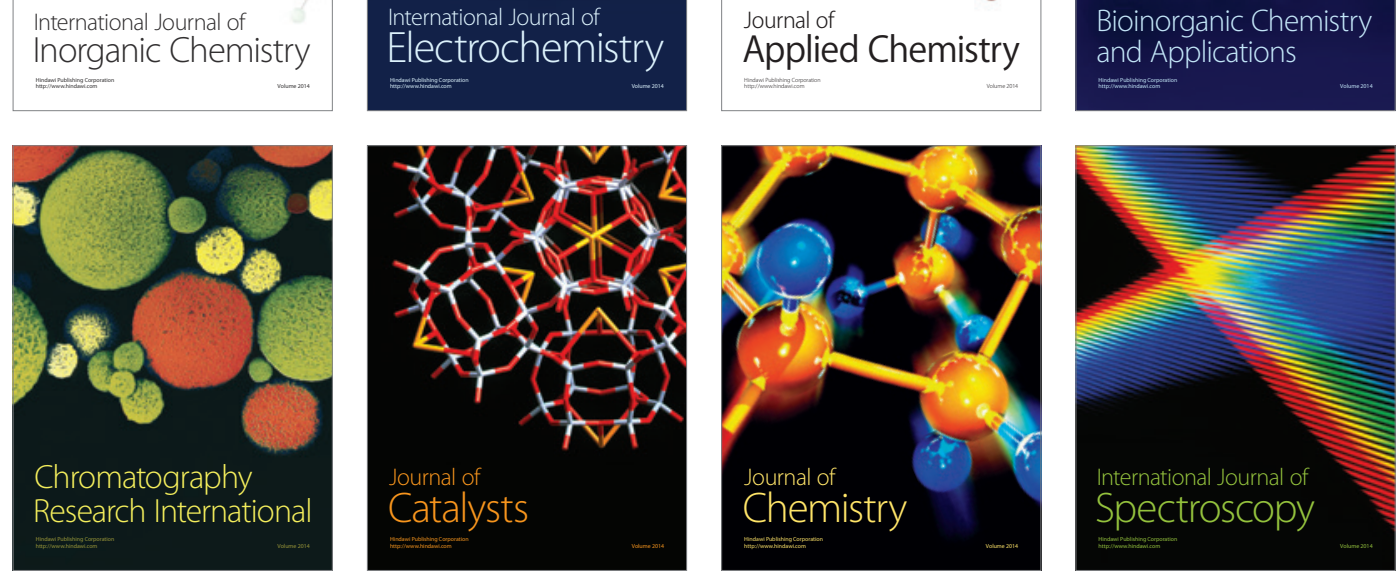